\title{
6
}

\section{The role of Benchmarking in the Management of Change process Some reflections from the TIME GUIDE project}

\author{
J. COLOM ${ }^{a}$, R. SMEDS ${ }^{b}$, S. KLEINHANS'c ${ }^{c}$ G. DOUMEINGTS ${ }^{c}$, M. BITTON ${ }^{a}$ \\ a CLEMESSY SA \\ 18 rue de Thann, BP 2499, 68057 MULHOUSE cedex FRANCE \\ b Faculty of Industrial Management, Helsinki University of Technology \\ Otakaari 4 A, 02150 ESPOO FINLAND \\ c GRAI/LAP University of Bordeaux \\ 351 cours de la Libération, 33405 TALENCE cedex FRANCE
}

\begin{abstract}
:
The use of benchmarking in the management of change is discussed, based on the approach of the EUREKA project TIME GUIDE.

Management of change begins by an essential process: the definition of the target. While it might seem trivial, this process, which needs clear references coming from the outside of the company, is often neglected.

A market study realized during the TIME feasibility phase illustrates this shortcoming in many ways.

In the ongoing project TIME GUIDE, a coherent methodology is developed for the management of enterprise evolution. This methodology contains, as an important part, the benchmarking method and database.
\end{abstract}

\section{Keywords :}

Management of Change, Steering the Evolution, Strategic Planning 


\section{INTRODUCTION}

For almost a decade, modifications in the economic and industrial environment have happened at such an increasing pace that traditional approaches of Manufacturing could not handle them. New models, new ways of thinking are required. In this context, industry as well as academic research pay more and more attention to such concepts as Management of Change including Benchmarking.

Benchmarking is a technique for increasing the knowledge of best practices. It is aimed towards managers wanting to evaluate their own level of performance, according to the achieved best level.

We can ask the question: what are the real objectives of managers using benchmarking?

- Is it to copy what is made by competitors, or even by non competitors in related industries?

- Is it to know what are the weakest points in their own companies, in order to motivate people, working in this area, to enhance their competitiveness?

- Is it on the contrary to know what are the company's strengths, in order to base their competitive strategy on them?

Perhaps all these reasons, and some others, could have a part in the decision of using benchmarking techniques. But the common point within these reasons, is the need to define some direction where the company has to go in its evolution process: Benchmarking helps to define strategic objectives, in order to steer the evolution and not to suffer it. It is then an essential step in the more global process of management of change.

Internationally, many projects in this field are emerging: "Agile Enterprise", "Intelligent Manufacturing Systems", etc.

In Europe, such programs as ESPRIT and EUREKA have been created in order to allow a common reflection of both industry and research at a European level, by launching precise projects ending in innovative technologies or management tools.

A group of European industrial and academic partners from Finland, France, Ireland, Italy, Norway, Portugal, Spain and Sweden prepared and proposed an Eureka Project, TIME (Tools and methods for the Integration and Management of Evolution of industrial enterprises). The project aims at developing a methodology and the supporting tools for steering the evolution process of industrial enterprises. Benchmarking is included in this methodology as an important part.

To be able to conduct a thorough market analysis before the specification of the project, it was decided to divide the project in two phases :

- the feasibility study;

- the project itself, called "TIME GUIDE".

This paper gives an overview of the TIME project:

- in the first part, we describe the past feasibility phase of the TIME project (from November 1992 to July 1993) as well as its key results;

- in the second part, we present the TIME GUIDE project stemming from the feasibility phase with special focus on the integration of benchmarking in the overall TIME methodology. 


\section{RESULTS OF THE TIME FEASIBILITY PHASE: THE MARKET STUDY}

\subsection{Organisation of the study [Time 93-2]}

During the TIME Feasibility Study in 1993, a user requirements survey was conducted in the following participating countries: Finland, France, Ireland, Italy, Norway, Portugal, Spain and Sweden. In the survey, industrial enterprises were asked about the evolution needs in their different functions and processes, and about the management of this evolution. Altogether 64 companies answered to the survey.

The targeted enterprises were manufacturing companies in various sectors mainly in automotive industry, machinery and electronics. For each of them, the contact person was a high level manager who had an overall view of the enterprise and could thus fill out most parts of the questionnaire alone.

We developed a "lean" questionnaire which could be completed in no more than two hours. The questionnaire was based on the Norwegian TOPP questionnaire [TOPP 92]. It was divided in three parts:

- The first part is an evaluation of the main functions' performance, compound of approximately 100 questions, each of them split up into 3 elementary sub-questions (performance today, performance in two years, importance for competitiveness). This allows to make projections towards the future, so that trends can be found.

There are 3 types of questions:

- overall performance of the function,

- level of computerization,

- additional specific questions.

- The second part is related to the practices and interests of the surveyed companies concerning their management of change (strategic issues, use of consulting, etc.).

- Finally, the last part contains background information about the company (name, size, sector, etc.).

The translated lean questionnaires were accompanied by documents explaining the context and objectives of TIME project to motivate the companies to participate to this survey. Each partner distributed this document to companies corresponding to the profile of study. To enhance the return ratio, each partner used its own contacts through the industrial milieu to convince most of them to respond.

A specific software application was developed to support the statistical analysis of the returned questionnaires. To validate and interpret the results further, also a more detailed TOPP analysis at five industrial sites was carried out.

\subsection{Main results [Time 93-2bis]}

The market study showed that most of the surveyed industrial companies have difficulties to define clear objectives in terms of strategic evolution. Indeed, while $77 \%$ of the enterprises claimed to have a strategic plan, only $64 \%$ had well defined goals and $46 \%$ had a clear definition of improvement projects in the strategic plan. This points out important shortcomings in the definition of a global strategy for evolution. 
Moreover, the companies in the recorded sample do not have methods to help them to define this strategic plan: only $25 \%$ of companies had knowledge about methods and tools for the management of evolution.

However, the consciousness of the importance of a long term vision and of a global reflection is very high. A long term view and management methods are recognised in the companies as key success factors for the evolution process. Nevertheless, they are not considered as sufficient by themselves: without a high motivation of employees, it seems difficult to successfully re-organise a company. Motivation is best achieved through participation. Therefore, it is a major key success factor to achieve a large participation during the change process.

\subsection{Functions needing major improvements}

According to the results, the greatest need for development in almost all functions was felt in small and medium sized enterprises with less than 500 employees. In general, they lagged behind the big companies in their relative performance. However, the managers of SMEs were aware of their huge need for development.

In those functions that were closely related to the physical system (the manufacturing and assembly functions, key equipment and manufacturing technology), the SMEs felt more competitive than the big companies. In logistics, irrespective of company size, the proximity of the company to the final customer seemed to coincide with higher performance estimates. In contrast to the big companies, the SMEs were however not yet aware of the whole logistic chain and of the importance of suppliers to overall competitiveness.

On the whole, SMEs have been so far focusing on the internal functioning and especially on manufacturing, which they now estimate to be very well equipped and competitive. No big development needs were felt in manufacturing, although quality and lead-time were perceived as important competitive factors. The respondents seemed not to associate development in quality and expedition of work processes with the development of their manufacturing function. The potential for organisational evolution in manufacturing needs still to be uncovered.

The function with the poorest ranking in performance relative to competitors was marketing. However, the perceived development needs in marketing were high. Also the rather low level of computerization in marketing was estimated to rise in the future. The enterprises perceived a need to improve their market knowledge and to react more quickly in order to win the hard competition.

In the researched sample of enterprises, there were problems in the integration of marketing with both production and quality. Also the maintenance function had poor links to quality and to design. Many of the enterprises could not estimate their ability of inter-functional cooperation at all. This is alarming, since the most important areas in evolution often are in the reintegration of differentiated tasks and functions into a more streamlined and efficient process.

Concerning the various functions' level of computerisation, attitudes are often ambiguous:

- the overall importance of computerisation for the competitiveness of the enterprise is not recognised,

- for many separate functions however, computerisation is depicted as very important, and moreover, its utilisation will increase in the next years.

Indeed, the strategic potential of IT is still uncovered, and the introduction of IT in industrial management has not yet included the necessary changes in the modes of operation: in work 
processes and organisation. There is in future a need for training and reorganisation in all business processes to take the full advantage of the already existing technology, and especially to implement most efficiently the huge future IT-investments that are anticipated in the answers.

\section{THE TIME GUIDE PROJECT}

\subsection{Objectives and Domain [Time 93-1]}

Accelerating turbulence in the competitive environment forces management to focus on mastering the continuous evolution of the enterprise. However, the methods and tools for this management of change are still insufficient. As a consequence, industrial organisations have evolved slowly and painfully. The evolution know-how has not accumulated in the organisation, and the enterprises' internal innovation potentials for evolution could not be uncovered. The results have been resistance to change, long lead times of transitions, often sub optimal techno-organisational structures and decreasing competitiveness.

TIME GUIDE aims to develop a set of tools for the enterprises' management of change needs. In TIME GUIDE, the evolution path of the enterprise is seen as a succession of intermediate steps - steady states - towards the vision "should be" (c.f. Figure 1). The management of evolution starts from the modelling and analysis of the present situation "as is" and the definition of a future vision "should be", and proceeds then to the modelling of the "next step", which will be the first intermediate steady state towards the vision. These managerial actions are repeated at each "next step", when it becomes the next steady "as is" on the path of evolution. Also the vision itself can get revised at each new "as is".

This continuous evolution through discrete steps has to be monitored and documented effectively, so that the organisation gets feedback from its progress along the path and can learn from its evolutionary actions. The definition of measurable progress factors and the establishment of evolution databases to create an "organisational memory of evolution" are thus important tasks in the management of change.

The self-audit method serves the internal diagnosis of the enterprise in its "as is" state, and contributes to the definition of progress factors. It helps to find the business processes that need to be developed, and to monitor this development. It is closely connected to the benchmarking method, that contributes to the external comparisons between enterprises on process level. The benchmarking results have an impact on the vision "should be": they create the tension for the change and help in the definition of the key performance indicators and the progress factors. An important feature in both the self audit and the benchmarking methods is their participative nature: both analyses are conducted by the personnel of the business processes and create thus commitment to change.

The TIME GUIDE model for the continuous development of enterprises is depicted in Figure 1 below. 


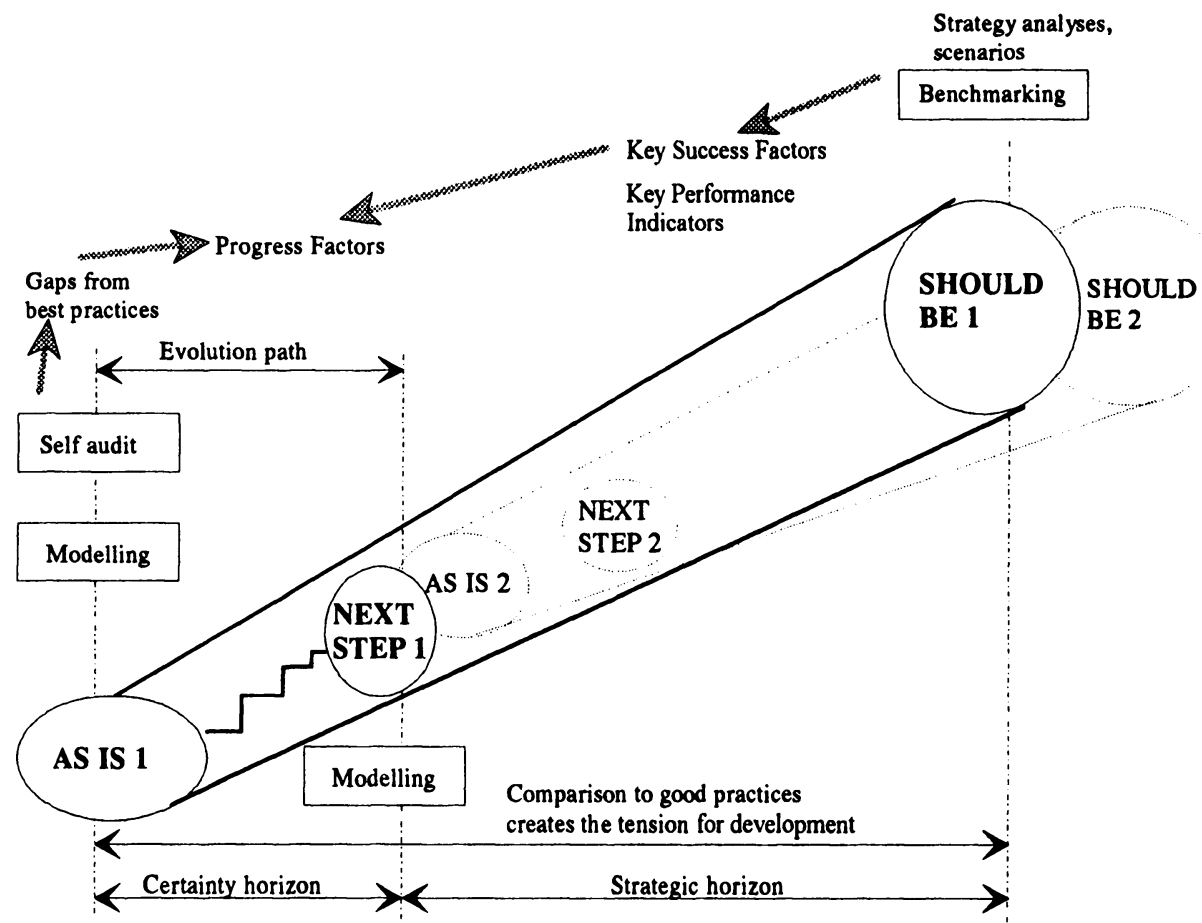

Figure 1: The TIME GUIDE model: Guiding the development of industrial enterprises [Time 93-3]

\subsection{Benchmarking in TIME GUIDE}

We would like here to show where and how benchmarking can be used in the overall change process and how the "classical" view of benchmarking can be enlarged and enriched by additional concepts and tools from TIME GUIDE. 


\subsubsection{Need of tools for benchmarking}

Today, benchmarking has reached a stage where everybody agrees more or less on the different steps in the process. Figure 2 hereafter sums up the benchmarking process.

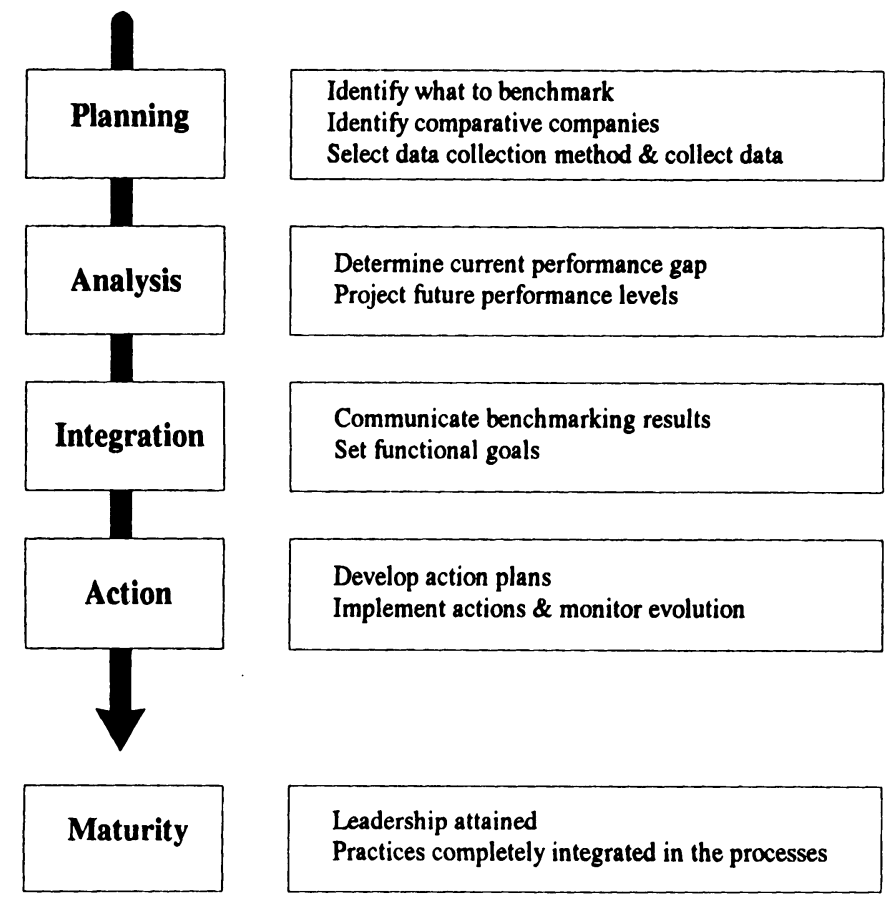

Figure 2: Steps in the benchmarking process [CAMP 89]

Yet, even if the benchmarking tasks are perfectly defined in their functional contents, the tools to support these tasks are missing: today, benchmarking proposes a process, but it is not enough founded on concrete implementation supports.

To illustrate this point, a 1992 study (in [Sprow93]) indicates that $95 \%$ of American companies do not know how to steer the benchmarking process, even if they are totally convinced of its necessity for the company.

For instance, few tools exist to help "benchmarkers" in the identification of the activity to benchmark. Most authors agree on the fact that this identification should be based on both customer's expectations (the "Order Winning Criteria") and strategic objectives of the company, but none of them proposes a clear methodology to steer this phase.

This is the case for most steps in the overall benchmarking process: how might companies determine the "Best Practices", how can they ensure their personnel's involvement, how can they measure the attainment of the functional goals they have set?

TIME GUIDE intends to provide such tools, and tentative solutions are already proposed : 
- by applying self-audit techniques, TIME GUIDE grounds the benchmarking process on a strategic diagnosis of the company's strengths and weaknesses allowing to define "those activities that contribute in providing competitive advantage" ([Shetty 93]). Moreover, a self-assessment tool might increase participation within the company, facilitating the final integration of the benchmarking results.

- by using modelling methods and tools, TIME GUIDE ensures that all aspects will be taken into account: the system is seen along decisional, informational, physical as well as organisational and human points of view.

The models and data obtained can not only help in identifying the key activities and processes to benchmark, but also:

- in building "generic models" of the benchmarked activity. These models would contain the key elements of a process (objectives, key resources and skills, associated performance indicators, flow of material, information, division of tasks etc.) and could help in finding the right comparative companies.

- $\quad$ in providing visual support (and ensuring a common understanding) to communicate internally the benchmarking results.

- $\quad$ in implementing the change, through the basic principle of participation in modelling and simulating the business processes.

- $\quad$ in documenting the change process. By updating periodically the models, the change actions and achieved improvements can be visualised, and an "organisational memory" of evolution can be created.

\subsubsection{The role of benchmarking in the management of change}

Management of Change is a broader concept than benchmarking: benchmarking is only one of the possible ways to tackle Management of Change. As such, benchmarking is included in the overall TIME GUIDE approach. We shall see hereafter where it takes place in the TIME GUIDE model of evolution: (c.f. Figure 1)

- The main role of benchmarking in our approach is to contribute, in addition to the more general strategic goals of the company, to the definition of the vision we have of the right direction to follow (the "Should be" system). By identifying "Best Practices", we can further determine the "Key Success Factors" leading to excellence in the considered business processes. Obtaining the same level of performance in our company becomes the target to reach, the objective initiating the whole improvement process.

- A second role of benchmarking lies in the determination of the "Next Step" as well as of the "action plans" to get there. By taking into account the situational factors and other "boundary conditions" of the benchmarked activity within our company (for instance, "allocated budget is limited to $1 \mathrm{M} \$$ ", or "Human resources policy forbids any hiring for the next two years"), we can also instantiate generic Key Success Factors and Key Performance Indicators into tangible progress factors leading to directly implementable action plans and measurable results. 


\subsubsection{Similarities between the two approaches}

The TIME GUIDE project is built in order to develop "Tools and methods for Integration and for Management of Evolution of industrial enterprises" that will take into account the industrial needs collected in the user requirements survey performed during the feasibility study.

The TIME GUIDE key concepts seem to fit well into the benchmarking approach and vice versa :

\section{Similarity 1 - TIME GUIDE as well as Benchmarking aim to steer the evolution process. \\ As a matter of fact, the objective of the two approaches is to improve the performance of the firm. The only difference lies in the improvement scope: while TIME GUIDE intends to manage the whole range of changes (structural, organisational, etc.) in an integrated and coherent approach, benchmarking focuses on precise improvement projects.}
Similarity 2- TIME GUIDE and Benchmarking imply participation and autonomy.
The two concepts are closely linked : autonomy is given prominence in order to enhance personnel's motivation and to reduce the resistance to change. Participation is therefore a sine qua non condition for the change process to be successful. Benchmarking insists on their importance, and TIME GUIDE proposes to embed these principles into all its tools and methods.

Similarity 3 - TIME GUIDE methodology and Benchmarking have to be applied continuously.

Most of the time, several improvement actions have to be planned, but also monitored and reviewed simultaneously. Results of these change actions have to be checked permanently, in order to keep coherence in the overall system. Moreover, changes occur permanently in the environment of the system. For adaptation purpose, continuity is required in steering the evolution process.

Similarity 4- Both TIME GUIDE methodology and Benchmarking take into account the competitive environment.

Both approaches end in durable, structural changes in the production system.

That is to say that both approaches deal with strategy, matching interna! strengths and weaknesses against the characteristics of the competitive environment.

This is not an exhaustive list of the similarities between TIME GUIDE methodology and Benchmarking, but it shows that both of them are grounded on the same conceptual framework stemming from - and aiming to solve - the difficulties encountered by industry in managing their change. 


\subsection{The European database for Benchmarking}

One of the aims of the TIME GUIDE project is to develop the structure for a European benchmarking database. The database will be built on the results of the TIME GUIDE benchmarking method, and filled with initial data from the TIME GUIDE pilot companies and companies participating in the Norwegian TOPP program. In TIME GUIDE, the feasibility of this database approach is proven, and a structure is settled for its future commercialisation and operational exploitation, which will be tasks of a following project.

\subsubsection{From self audit to benchmarking: experiences from the TIME feasibility study}

In five companies of the participating TIME countries, an in-depth industrial site analysis was carried out during the feasibility study. Researchers in the TIME consortium collected the data from the sites using the full TOPP A questionnaire as an instrument. The aim of these analyses were firstly to test the results of the "lean" market study, and secondly to test the self audit and benchmarking features of the TOPP A approach. The results were analysed at SINTEF using the TOPP method and the already existing TOPP database.

The TOPP A questionnaire is formulated as a self-assessment of the company's own performance compared to the performance of its competitors. Performance estimates are given for each function of the company in its present situation and in the estimated situation in two years. All organisational functions are assessed in principle two times: on a general level by around 20 individual respondents from different units of the organisation, and on a more detailed level by small interdepartmental groups. The assessments are given as relative scores (1...7) of own performance compared to the competitors' performance. Also the importance of each function to the company's competitiveness is evaluated.

The development needs of the five industrial sites could be analysed in a number of ways. The group results highlighted the functions which had biggest gaps between the present performance and the estimated performance in two years. They could also point out the critical development areas, where the importance for competitiveness was assessed to be very high but the present performance was ranked poor. On the other hand, some functions could be detected, where big development needs were expressed, but which were not considered important for competitiveness. The individual responses revealed those functions where the differences of opinion concerning performance were the greatest. This could be interpreted as a sign for development needs in those functions.

\subsubsection{Requirements for the TIME GUIDE benchmarking database}

The TOPP A self audit results can help companies to find, based on internal self-assessment, their most critical functions and processes. When conducted as a continuous self audit activity, the results also show development trends within one company. But the audit scores as such are meaningless for inter company comparison. They are given by the companies themselves as subjective estimates of own performance relative to competitors. For benchmarking, these self images should be complemented by objective measurement data, and a common process modelling structure.

The structure of the self audit hierarchy of measurement criteria will in the TIME GUIDE project be mapped with the structure of the benchmarking database, and links will be established to enable inter company comparison. The objective is to develop a benchmarking procedure and database structure, that allows company comparisons on a process level. A deep 
benchmarking in the relevant areas of enterprises (in their business processes, in manufacturing, logistics etc.) by the managers and middle managers themselves in a participative manner gives important knowledge about how these enterprises reach their performance levels (not only their performance levels as such).

The TIME GUIDE industrial partners as well as selected TOPP companies will test the benchmarking method and the database prototype in pilot implementations, cooperating with project researchers. Based on this cooperation, pilot cases describing the experiences and results from the benchmarking method will be produced. These cases will later on help further refining the database structure before the final version is developed.

\section{CONCLUSION}

According to the market survey conducted in the TIME feasibility study, industrial managers feel huge development needs in their companies, and a well managed enterprise evolution is perceived as a competitive advantage. The demand for effective change management tools and methods has awakened and is growing rapidly.

To answer to this need, the TIME GUIDE project will develop a methodology for change management. The TIME GUIDE methodology and benchmarking are closely connected and complementary in at least three dimensions:

- $\quad$ TIME GUIDE fulfils the need for tools that support benchmarking by developing a self audit procedure and modelling techniques

- Benchmarking is perfectly integrated into the overall TIME GUIDE methodology, since it helps in determining the goals to strive for (the "should be" system and the "next steps"); it also helps in the formulation of the progress factors to measure the concrete change actions.

- The objective (improvement of enterprise performance) as well as the underlying concepts (continuity, participation, consideration of the competitive environment) are common to both approaches.

The TIME GUIDE methodology consists of three separate methods: the self audit method, the modelling techniques and benchmarking. All these elements answer to specific requirements in evolution management. Considered separately, each of them has strengths and weaknesses. But built into a coherent TIME GUIDE methodology, the synergy and complementarity of the elements make the whole more than the sum of its parts.

While benchmarking alone is not sufficient to manage enterprise evolution, its integration into the TIME GUIDE methodology leads to a coherent management of change process. The central effort in the TIME GUIDE project will lie in developing this synergy between the three methods. 


\section{BIBLIOGRAPHY}

[Camp 89]

[Shetty 93]

[Sprow 93]

[TOPP 92]

[Time 93-1]

[Time 93-2]

[Time 93-2bis]

[Time 93-3]
Robert C. Camp, "Benchmarking - the search for Industry Best Practices that lead to superior performance" - ASQC Quality Press 1989, Milwaukee, Wisconsin.

Y.K. Shetty, "Aiming high: competitive benchmarking for superior performance" - Long Range Planning, Vol. $26 \mathrm{Nr}$ 1, pp 39-44, Pergamon Press Ltd, 1993.

E.E.Sprow, "Benchmarking: it's time to stop tinkering with manufacturing and start clocking yourself against the best" - Manufacturing Engineering, pp56-69, September 1993.

TOPP "A productivity program for manufacturing industry" - NTNF 1992.

TIME -deliverable D11: "Objectives, concepts and domain of the TIME project". Last version - 4/10/1993.

TIME -deliverable D21: "Suney methodology". Last version - 6/10/1993.

TIME -deliverable D22: "Results of the market survey". Last version $6 / 10 / 1993$

TIME -deliverable D31: "Definition of the deliverables". Last version 4/10/1993. 